\title{
Infrared emissions from shock heated hydrocarbons
}

\author{
K. M. Stephens and S. H. Bauer \\ Baker Chemical Laboratory, Cornell University, Ithaca, NY 14853-1303, U.S.A.
}

(Received 28 January 1993; in final form and accepted 9 May 1993)

\begin{abstract}
The primary objective of this study was to ascertain whether low molecular weight hydrocarbons (LMWH) in the range $C_{4}$ to $C_{7}$, upon heating to temperatures above $900 \mathrm{~K}$, cmit IR radiations at frequencies that correspond to the "unidentified infrared" (UIR) features-the recorded emissions from a variety of astronomical sources-reflection nebulae, HII regions, planetary nebulac, spiral galaxies and other extra galactic objects. We describe IR emission spectra recorded from shock-heated gases $\left[\mathrm{C}_{2} \mathrm{H}_{2}:\left(\mathrm{H}_{3} \mathrm{C}\right)_{2} \mathrm{C}=\mathrm{CH}_{2}\right.$; $\left.\mathrm{H}_{2} \mathrm{C}=\mathrm{C}\left(\mathrm{CH}_{3}\right)-\mathrm{C}\left(\mathrm{CH}_{3}\right)=\mathrm{CH}_{2} ;\left(\mathrm{H}_{3} \mathrm{C}\right)_{2} \mathrm{C}=\mathrm{CH}-\mathrm{C}_{(}\left(\mathrm{CH}_{3}\right)=\mathrm{CH}_{2}\right]$, that arise from excitation of the fundamental $\mathrm{C}-\mathrm{H}$ stretching vibrations. While the IR emissions from $\mathrm{LMWH}$, anticipated over the entire spectra range, do not present a perfect match to UIR, the correspondence over several wavelength regions is better than the emissions anticipated from polycyclic aromatic hydrocarbon (PAH) species. Finally, we briefly review the range of proposals that have been presented for the origin of the UIR bands.
\end{abstract}

\section{INTRODUCTION}

THE EXPERIMENTS described below were initiated to obtain answers to several questions:

(i) Are the recorded but unidentified IR emissions from a variety of astronomical sources (UIR) due solely to polycyclic aromatic hydrocarbons (PAH), or should one also consider additional plausible candidate species, in particular selected low molecular weight hydrocarbons ( $\mathrm{LMWH}$ )? Astrochemists generally agree [1] that no single Earthbound substance has heretofore been proposed that matches all the observed features in wavelength and intensity, and concurrently does not indicate additional strong features that are not observed, yet the PAH hypothesis holds sway.

(ii) Since the IR absorption spectra of selected alkenes and dienes match overall the astronomical radiations about as well as do the (approximately) calculated emission spectra of PAH (see Fig. 1), would the IR emissions from hot LMWH show a comparable match, so that conventional absorption spectra may provide useful guides?

(iii) Can one record IR emission spectra from heated LMWH prior to their pyrolytic decompositions?

\section{RATIONALE FOR OUR EXPERIMENTS}

If one grants the possibility that LMWH known to be present in significant densities in regions characterized by equivalent temperatures $T>1000 \mathrm{~K}$, are emitters of UIR bands, then it should be possible to demonstrate the production of such spectra experimentally. Restated, under what conditions of temperature and pressure do thermally excited emissions from LMWH, in the IR, show spectroscopic signatures that match their IR absorption spectra? An essential condition is that the emissions be recorded under homogeneous conditions at low gas densities, prior to their pyrolysis. This can be done by utilizing shock wave heating.

Before presenting some details of our experimental protocol [2] it will prove informative to list briefly the advantageous features and the limitations of the shock tube technique. Foremost, heating is accomplished by gas dynamic pressure waves while the tube walls remain at room temperature. The test gas is highly diluted in an argon carrier $(\approx 1 \%$ level). With rapid response pressure devices and optical measurements one can determine both the temperature and the density of the test gas under shock tube conditions [3]. For the device shown in Fig. 2(a, b) the accessible range of experimental temperatures is $800-4000 \mathrm{~K}$. However, the following limitations apply: (i) the inert gas is an essential component of the shocked sample; and (ii) the heating pulses are of short

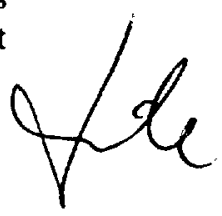



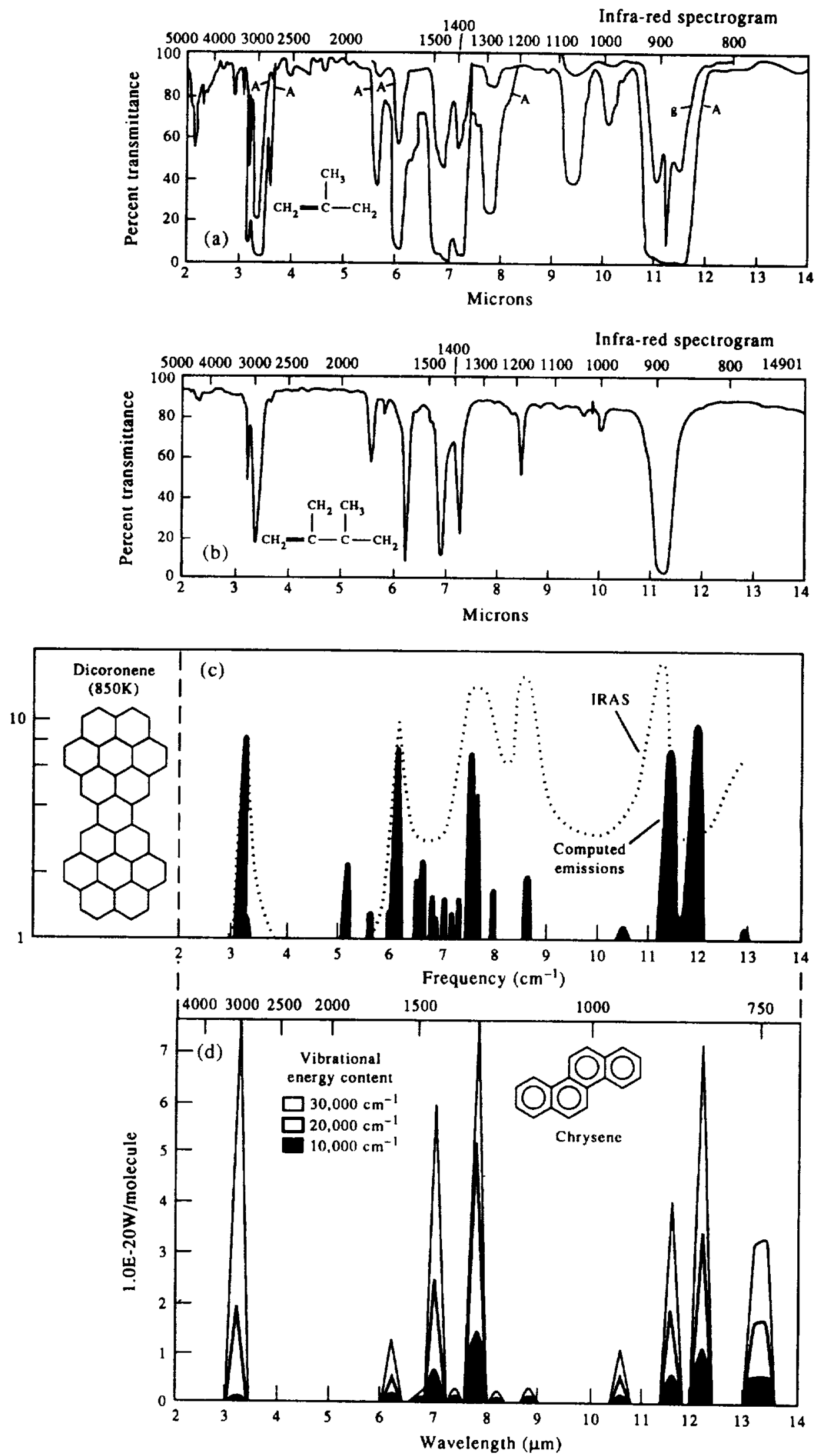

Fig. 1. Comparisons of recorded absorption spectra of 2-methyl-propene (a) and 2,3dimethylbutadiene (b) (gas phase) with IRAS, and IR emissions computed for dicoronene (c) (at $850 \mathrm{~K})$ [19], and fluorescence from chrysene at three levels of photoexcitation [25] (d). 
duration- 3 to $6 \mathrm{~ms}$; therefore one must record emission intensity profiles rapidly, and is thus limited to a single IR wavelength for each shock. To develop a spectrum at successive spectrometer settings one must repeat shock-runs, which are intrinsically only approximately reproducible. With a 20 strip array detector (a thermoelectrically cooled $\mathrm{PbSe}$ array) the required number of repeat runs may be reduced by the same factor. Unfortunately, the unit we acquired was low in sensitivity, noisy and of poor reproducibility. Hence almost all of our data were recorded with an InSb liquid nitrogen cooled single-slit detector, set at a modest resolution: $\lambda / \Delta \lambda \approx 82$. The fidelity in reproducing absorption spectra is illustrated below.

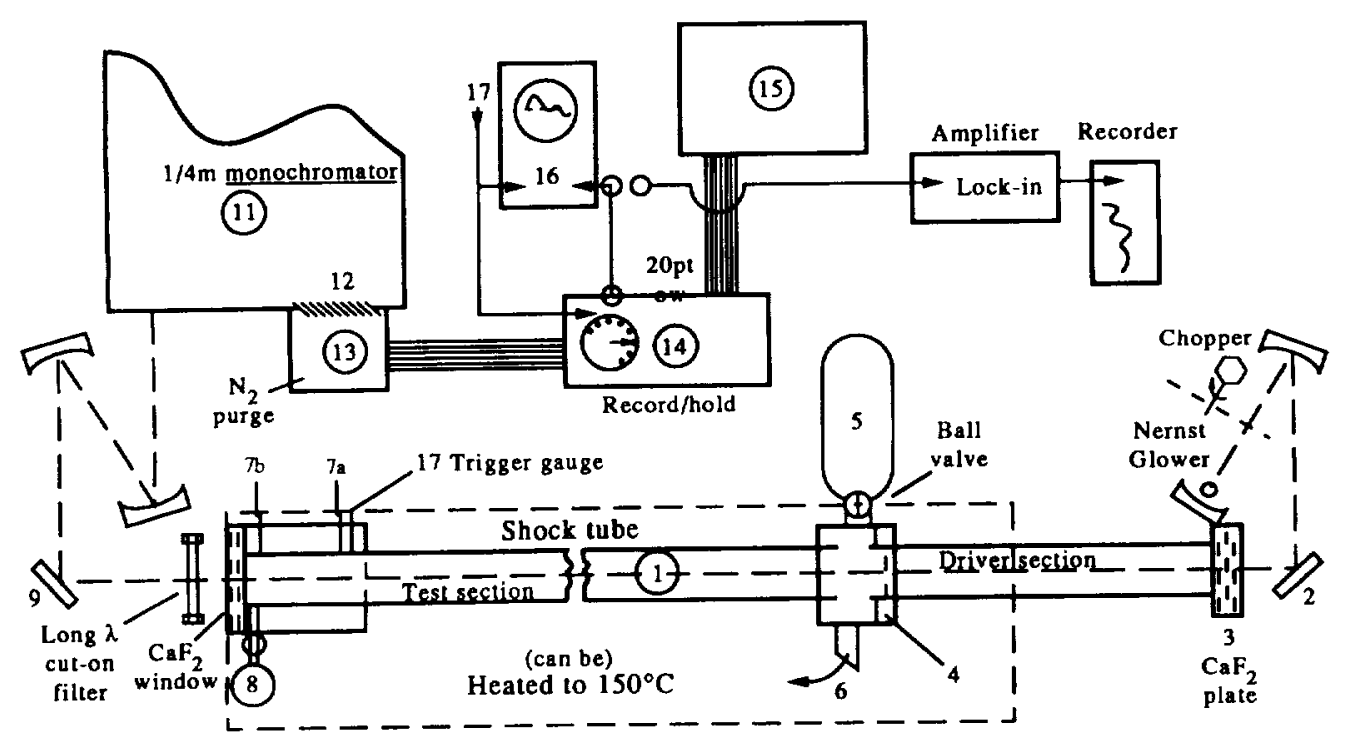

Key: (1) Shock-tube; 1" I.D. stainless steel.

(4) Diaphragm holder.

(5) Reflected shock damping tank (connected via large ball valve)-Runs were made both with the large-ball valve to tank (\#5) open (single-pulse operation), or closed (conventional operation). We found no significant difference in the recorded pressure profiles generated by gauges $7 \mathrm{a}$ and $7 \mathrm{~b}$ for the initial $6 \mathrm{~ms}$.

(6) Line to vacuum pumps; gas sample reservoir.

(7a. 7b) Piezo-electric pressure sensors ( $1 \mu$ s rise time).

(8) Sampling bulb for analysis of processed gas.

\begin{tabular}{lll}
\hline (11) Gratings & Blazed at & Resolution (at midrange) \\
\hline (a) $18001 / \mathrm{mm}$ & $0.5 \mu \mathrm{m}$ & $69.6 \mathrm{~cm}$ \\
(b) 1200 & 0.3 & 77.1 \\
(c) 600 & 1.5 & 33.6 \\
(d) 300 & 2.0 & 16.8 \\
(e) 150 & 4.0 & 16.7 \\
\hline
\end{tabular}

Total scan range: $450 \mathrm{~nm} 106.5 \mu \mathrm{m}$.

(12) 20 Element array of radiation sensors.

(13) Low noise amplifiers-parallel processing.

(12/13) Alternate: replace by a single $1 \mathrm{~mm}$ wide slit and an InSb LN2 cooled infrared detector.

(14) Digitizers for amplified 20 channels (and/hold)

(15) Computer and printer.

(16) Oscilloscope for monitoring and photorecording from InSb detector, or from any selected element; second beam-for amplified pressure signals. Not shown: Signals from 7a, 7b are added and amplified; digitized in Biomation 8100, and sent to plotter; in addition, amplified pressure signals are sent to the oscilloscope.

Fig. 2(a). Experimental set-up. 
Protocol for Spectral Data Collection (A Brief Outline)

Prior to shock operation the entire tube was evacuated and the optical system calibrated by recording IR intensities (at a sequence of wavelength settings of the monochromator) that are emitted by the Nernst glower. Its temperature was measured with an optical pyrometer. Subsequent emission intensities were then ratioed to the transmissitivity/sensitivity function for the optical/electronic system, relative to the black-body curve that was calculated from the measured glower temperatures. Since only relative intensities were required for this study, correction for the glower emissivity function was unnecessary because $\varepsilon$ does not vary significantly over the wavelength range we investigated $(1.0-5.0 \mu \mathrm{m})$.

The shock tube, illustrated in Fig. 2(a) was swabbed after each run. A suitable mylar diaphragm was inserted and both sides were evacuated with a roughing pump. Then the (longer) test section was connected to the diffusion pump (pressure reduced to $\approx 10^{-5}$ Torr). After filling the test section (from the storage tank) with a mixture of approximately $99 \%$ argon plus $1 \%$ test gas, to a total pressure of $20-80$ Torr, the driver section was slowly filled with helium to a pressure of approximately $83 \mathrm{psig}$. Then the diaphragm burst and the shock wave was initiated. The oscilloscope and the electronic recording devices were triggered by signals that originated from pressure gauge \#17. The incident shock speed was read from the pressure records stored in the Biomation 8100 unit. Incident and reflected shock temperatures as well as corresponding densities were calculated therefrom, based on the sample pressure (as set up), and the heat capacity ratios for the test gas and the driver gas.

The heated sample, either in absorption or emission, is viewed along the tube axis. Note that with the axial configuration each slice of the sample (normal to the axis) is sequentially and rapidly raised to the shock temperature (in about $0.1 \mu \mathrm{s}$ ). The total

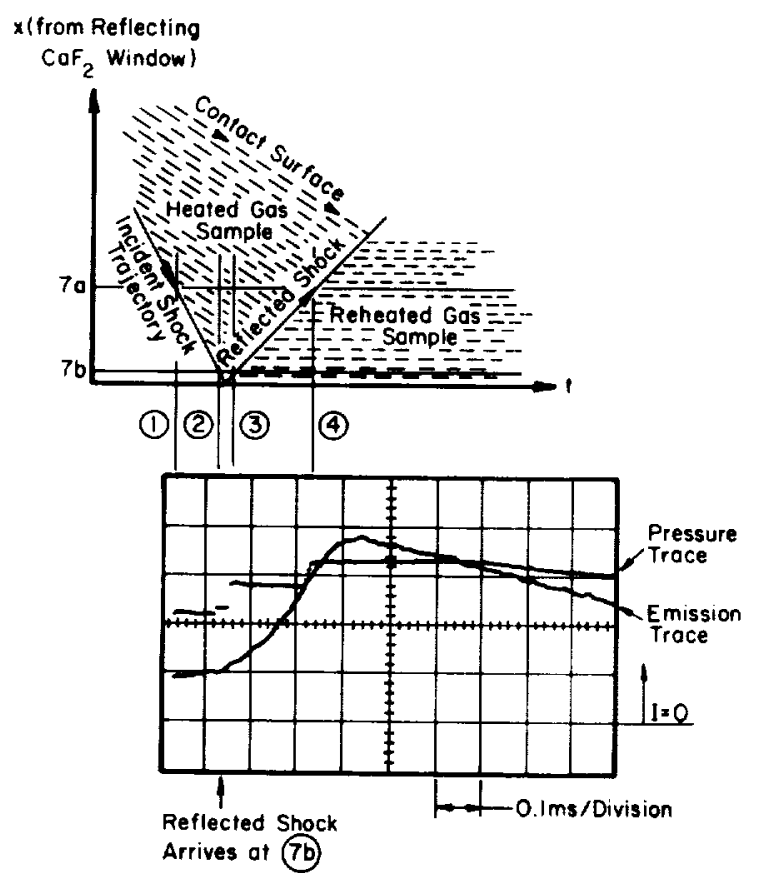

Key: The pressure trace is aligned with a wave-diagram that correlates shock speeds and arrival times at the shock tube terminus. The pressure sensors are connected in series. The sum of the output voltages shows an initial rise when the incident shock arrives gauge 7 a(1); then a second rise at $7 b(2$. After reflection at the end window, the reflected (reheating) shock arrives at $7 b$ (3). and later at $7 \mathrm{a}(4) .$. two large jumps.

Fig. 2(b). Typical oscilloscope traces, showing superposed signals from the pressure sensors and emission intensity over a $1.0 \mathrm{~ms}$ interval. 


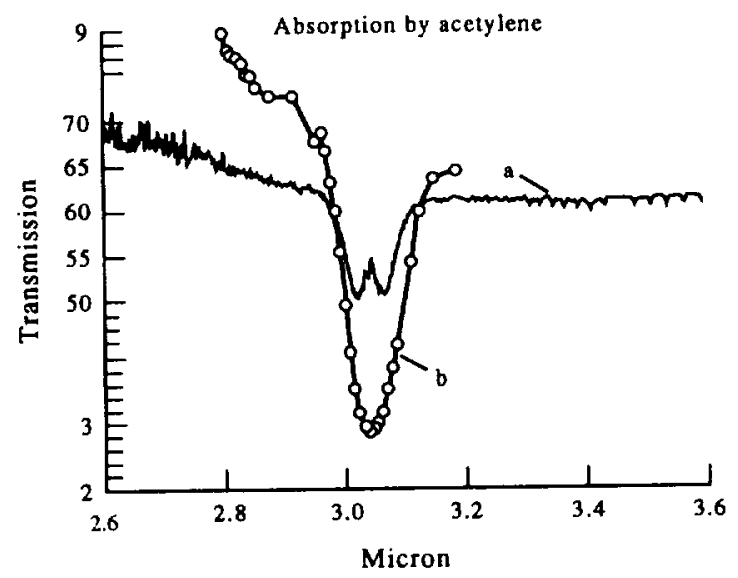

Fig. 3. Reference absorption spectra of acetylene. (a) Recorded on a Mattson FTIR, with $0.004 \mu \mathrm{m}$ resolution; (b) recorded with our $1 / 4 \mathrm{~m}$ monochromator, background corrected for black-body emission intensity.

sample viewed grows progressively with time, as the reflected shock moves away from the window, at a rate of $\approx 5.7 \times 10^{4} \mathrm{~cm} / \mathrm{s}$. At very short times, immediately on shock reflection, the sample is optically thin and becomes optically thicker as the shock recedes; it is ultimately quenched by the oncoming expansion wave. Typical conditions for these shock tube experiments were: gas composition: 1-5\% $\mathrm{H} / \mathrm{C}$, with $\mathrm{Ar}$ as the carrier gas; the driver gas was helium. Initial conditions: $p_{0}$ (total driven gas) = $20-40$ Torr; $p_{4}$ (driver pressure) $=30-50 \mathrm{psi} ; T_{0}=296 \mathrm{~K}$. Incident shock speeds ranged from $0.9-1.1 \mathrm{~km} / \mathrm{s}$. The shock traversal time between piezo-stations $[\Delta x(7 \mathrm{a}-7 \mathrm{~b})=100.0 \mathrm{~mm}]$ was measured to $\pm 0.2 \mu \mathrm{s}$. Typically for $1 \% \mathrm{C}_{2} \mathrm{H}_{2}, T_{2}=1.29 \times$ $10^{3} \mathrm{~K}$ (incident wave), and (depending on whether one assumes conversion) $T_{5}^{0}=$ $2.61 \times 10^{3} \mathrm{~K}$, whereas $T_{5}^{\mathrm{cq}}=2.56 \times 10^{3} \mathrm{~K}$ (in the reflected wave).

\section{InVESTIGation OF ACETylene (Test of Model)}

Using the 150 line/mm grating, in the $1 / 4 \mathrm{M}$ spectrometer, we calculated that with $1 \mathrm{~mm}$ slits the best resolution attainable would be $0.02 \mu \mathrm{m}$. However, our recording of the $v_{3}$ band of $\mathrm{C}_{2} \mathrm{H}_{2}$ at $3.04 \mu \mathrm{m}$ (Fig. 3) did not show the split between the $P$ and $R$ branch maxima; they appear in the absorption spectrum recorded with the Mattson FT spectrometer at a stated resolution of $0.004 \mu \mathrm{m}$, indicating that our experimental resolution was approximately $0.04 \mu \mathrm{m}$; this is clearly adequate for the present experiment.

The next concern was the survivability of acetylene at various reflected shock temperatures. Reference to the report by COLKET [4] (which is a representative report selected from a vast literature on the $\mathrm{C}_{2} \mathrm{H}_{2}$ pyrolysis) shows that only above $1800 \mathrm{~K}$, at dwell times of $0.70 \mathrm{~ms}$, is there any significant conversion of acetylene to diacetylene; but even at $2400 \mathrm{~K}$, the amount of $\mathrm{C}_{4} \mathrm{H}_{2}$ generated remains an order of magnitude lower than of acetylene (Fig. 4 for $3.7 \% \mathrm{C}_{2} \mathrm{H}_{2}$ in argon). Furthermore, when one starts with vinyl acetylene $\left(\mathrm{C}_{4} \mathrm{H}_{4}\right)$, the original species is pyrolyzed above $1800 \mathrm{~K}$ to the major product, which is acetylene; the ratio of acetylene to diacetylene [5] remains at a factor of 10 .

The spectrum we previously recorded with a silicon diode array showed the Swan System. Hence we looked for the $C_{2}$ emission band at $2.47 \mu \mathrm{m}\left[d^{3} \Sigma^{-} g-a^{3} \Pi_{u} ;(1-0)\right]$, when $\mathrm{C}_{2} \mathrm{H}_{2}(1 \%$ in argon) was shock heated to reflecting shock temperatures $1600-$ $3200 \mathrm{~K}$. No signals above background levels were observed. However, a structureless background of radiation, reaching a maximum level of about 1/3 of the characteristic $\mathrm{C}-\mathrm{H}$ spectrum was recorded in the reflected shock regime. A typical emission profile is shown in Fig. 5(a). Note that the background emission is initiated by the reflected shock; it begins to decline at $\approx 1 \mathrm{~ms}$, and approaches zero at about $1.75 \mathrm{~ms}$. The measured 


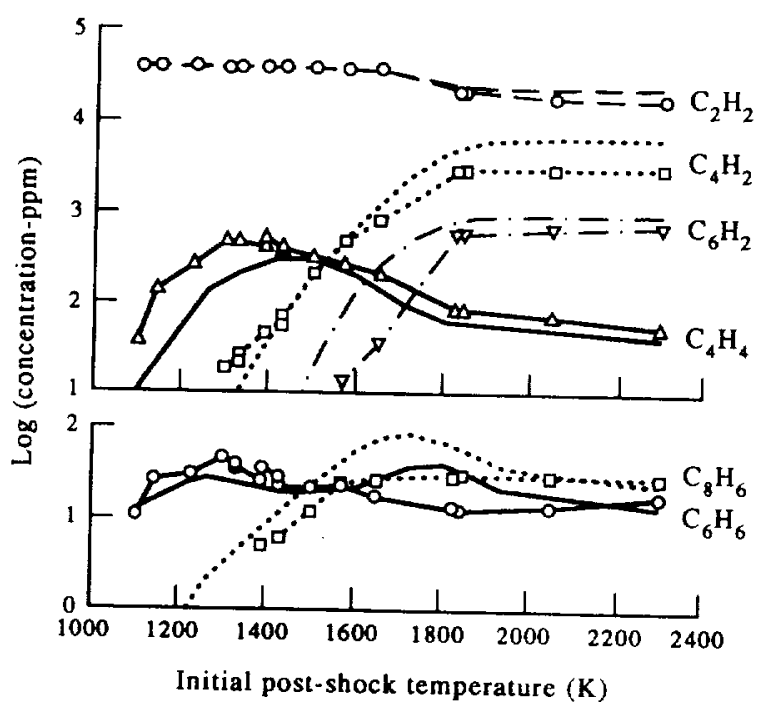

Fig. 4. Product distributions of shocked pyrolysis of acetylene, as a function of temperature (3.7\% in Ar). Dwell times $\approx 700 \mu$ s; data obtained by Col.Kt:I [4]. The solid lines connect experimental points; the dashed lines show molel calculations

maxima of this background radiation are listed in Table 1 . We checked for the appearance of soot due to pyrolysis. Very little was produced, hardly enough to show color on the cleaning cloth used to swab the tube between runs.

Overall, spectra were recorded for reflected shock temperatures from 1250 to $3750 \mathrm{~K}$. A typical response curve for spectrometer settings between 2.80 and $3.6 \mu \mathrm{m}$ is shown in Fig. 5(b). Note the significant difference between traces a and b; whereas the former starts at zero at the onset of the reflected shock, the latter shows $1 \mathrm{~cm}$ defection at the initiation of the oscilloscope trace, indicating that emission at this wavelength started during the incident shock. In Fig. 5(c) the time-span recorded was reduced from 0.5 to $0.1 \mathrm{~ms} / \mathrm{cm}$.

The temporal dependence of emission intensity on temperature is illustrated in Figs 6 and 7. At the lower temperature (reflected shock temperature $\approx 2350 \mathrm{~K}$ ) the maximum emission appears precisely at the same wavelength as was recorded in absorption. Its intensity declines with time as expected for a cooling medium. At early times there is a clear indication of a shoulder on the long wavelength side. In Fig. 7 (reflected shock temperature $\approx 2650 \mathrm{~K}$ ) the shoulder is more prominent, and in both figures the magnitude of the shoulder declines more rapidly than does the main peak at $3.04 \mu \mathrm{m}$. Vibrational assignments for $\mathrm{C}_{2} \mathrm{H}_{2}$ have been made by CIIILD and LAwTON [6]. The most intense IR transition arises from the asymmetric $\mathrm{C}-\mathrm{H}$ stretching vibration $[00000 \leftarrow 00100]$, centered at $3.034 \mu \mathrm{m}$. Next in intensity is $[10000 \leftarrow 10100]$, centered at $3.147 \mu \mathrm{m}$. The dependence of the relative populations in the originating 00100 and 10100 states on temperature is shown in Fig. 8. The emission from the $3.15 \mu \mathrm{m}$ band declines when the shocked medium cools. The relative intensities scale reasonably well with the corresponding gas densities, in the reflected shock regime.

The earliest emission that can be recorded is limited by the mean radiative lifetime of the emitter; in the IR it is $\approx 0.1-1.0 \mathrm{~ms}$. The observed maximum appears $\approx 160 \mu \mathrm{s}$ after initiation by the reflected shock. A simple mechanism reproduces the recorded temporal evolution of these emissions; it incorporates excitation and pyrolysis steps, and radiation loss from the body of heated gas as it propagates along the shock tube (see Appendix 1).

The above experiments (based on acetylene as a test species) demonstrated that emissions in the IR. developed by shock heating low molecular weight hydrocarbons, bear direct relations to the room temperature absorption spectra of the corresponding species; the temporal evolution of the emission spectra provide useful data on state populations, and thus impose constraints on mechanistic models. Furthermore, such spectra can be captured from shock heated samples for periods up to $5 \mathrm{~ms}$ after shock 


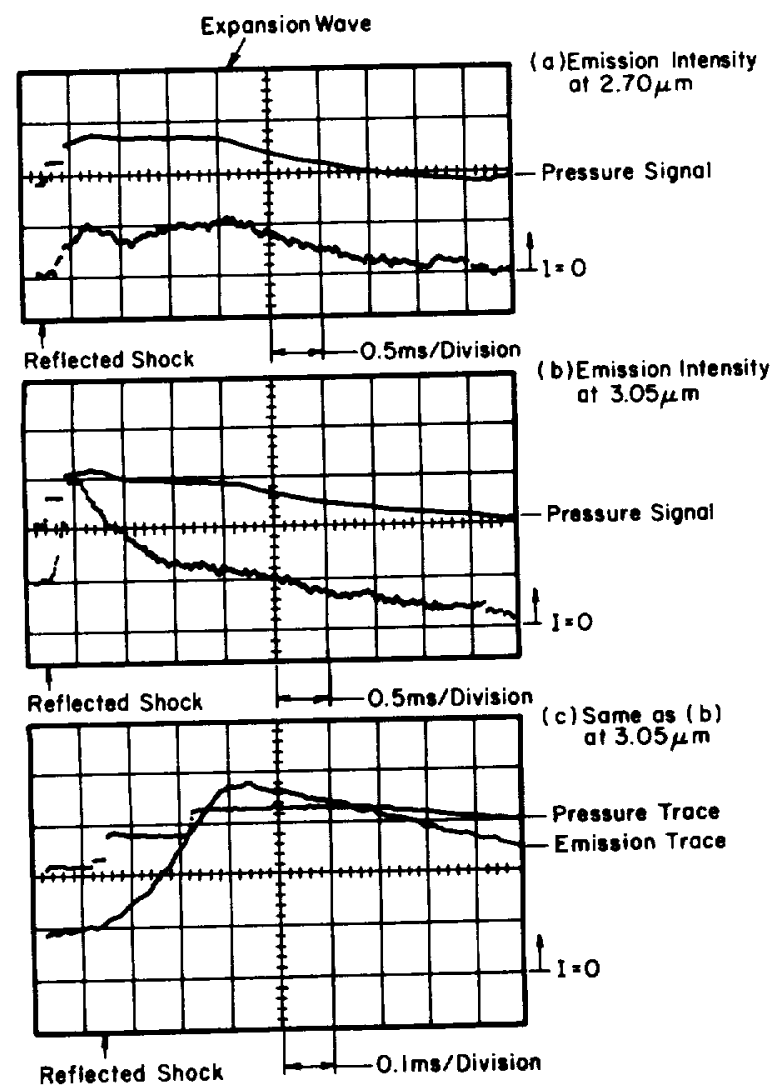

Fig. 5. Oscilloscope traces of shock pressure histograms, and IR intensities emitted by acetylene (a) at $2.70 \mu \mathrm{m}$ : background; (b and c), at $3.05 \mu \mathrm{m}$.

initiation and prior to extended pyrolysis. Our next objective was to demonstrate that emission spectra can be generated from the more fragile molecular species by shock heating at lower temperatures (starting at $\approx 900 \mathrm{~K}$ ).

\section{Emission Spectra from Methyl-substituted Alkenes}

\section{$\overline{\left(\mathrm{CH}_{3}\right)_{2} \mathrm{C}=\mathrm{CH}_{2}}$}

Spectra of $1 \%$ 2-methyl propene (in Ar), shock heated to $1400 \mathrm{~K}$, show as expected, a strong emission at $3.40 \mu \mathrm{m}$ (assigned to the $\mathrm{CH}_{3}, d$-stretch; $v_{25}$ with sym. $b_{2}$ ) and an overlapping (at our resolution) companion band at $3.24 \mu \mathrm{m}$ (assigned to $\mathrm{CH}_{2}$, a5-stretch; $\nu_{1 n}$ with sym. $\left.b_{1}\right)$. These are the two strongest bands in the $3-4 \mu \mathrm{m}$ region [7]. Inspection of Fig. 9 indicates that their relative intensity changes little, as both bands decline due to falling temperature of the emitter, as expected. The strong emission at $3.4 \mu \mathrm{m}$ rises

Table 1. Emissions from $\mathrm{C}_{2} \mathrm{H}_{2}$

\begin{tabular}{ccc}
\hline $\begin{array}{c}T_{5} \\
\text { (reflected shock) }\end{array}$ & $\lambda(\mu \mathrm{m})$ & $\begin{array}{c}\text { Relative } l \\
\text { (max) }\end{array}$ \\
\hline 16601 & 2.45 & 0 \\
26.50 & 2.45 & 1.0 \\
$32(0)$ & 2.45 & 1.4 \\
$32(0)$ & 2.60 & 1.2 \\
32001 & 2.70 & 1.0 \\
\hline
\end{tabular}




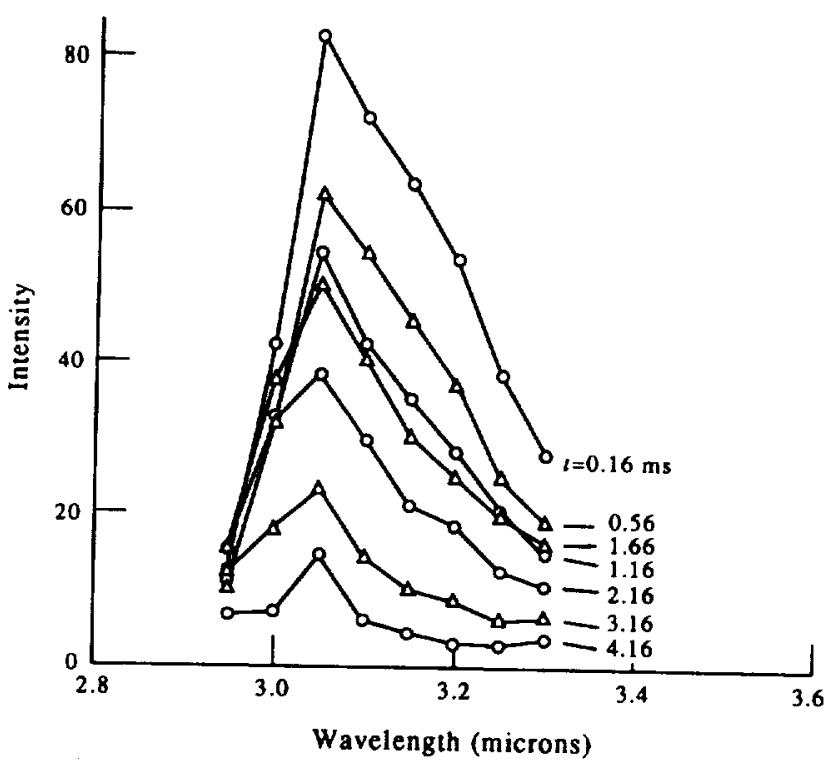

Fig. h. Time-dependent emission intensities recorded at a sequence of monochromator settings $(2.95 \rightarrow 3.30 \mu \mathrm{m})$, from $1 \%, C_{2} \mathrm{H}_{2}$ in Ar. shock heated to $2350 \mathrm{~K}$. Spectral resolution $\approx(1.04 \mu \mathrm{m}$. The indicated times were measured post arrival of the reflected shock at position (3): Fig. 2(b).

sharply and attains a maximum at $0.49 \mathrm{~ms}$; then it decays. As in the room temperuture absorption spectrum, another fundamental $\mathrm{C}-\mathrm{H}$ frequency but of weaker intensity, at $3.25 \mu \mathrm{m}$, appears as a shoulder on the higher frequency side of the strong band. The split became more apparent when these spectra were recorded at closer $\Delta \lambda$ intervals, but was not as deep as in the absorption spectrum, due to the broadened rotational structure of the two bands at the higher temperature. Emission starts at incident shock temperatures, recorded as the shock wave approaches the end window; then it rises sharply upon shock reflection. The maximum intensity at $3.4 \mu \mathrm{m}$ peaks at $\approx 1300 \mathrm{~K}$, reflected shock temperature. As it declines. IR emission at $3.04 \mu \mathrm{m}$ rises due to acetylene production, first at $1400 \mathrm{~K}$. Early single-pulse shock tube experiments with isobutene [8] indicated that the unimolecular rate constant for dissociation $\left(\rightarrow \mathrm{H}_{2} \dot{\mathrm{C}}-\mathrm{CH}=\left(\mathrm{CH}_{2}+\dot{\mathrm{C}} \mathrm{H}_{3}\right)\right.$ had an activation

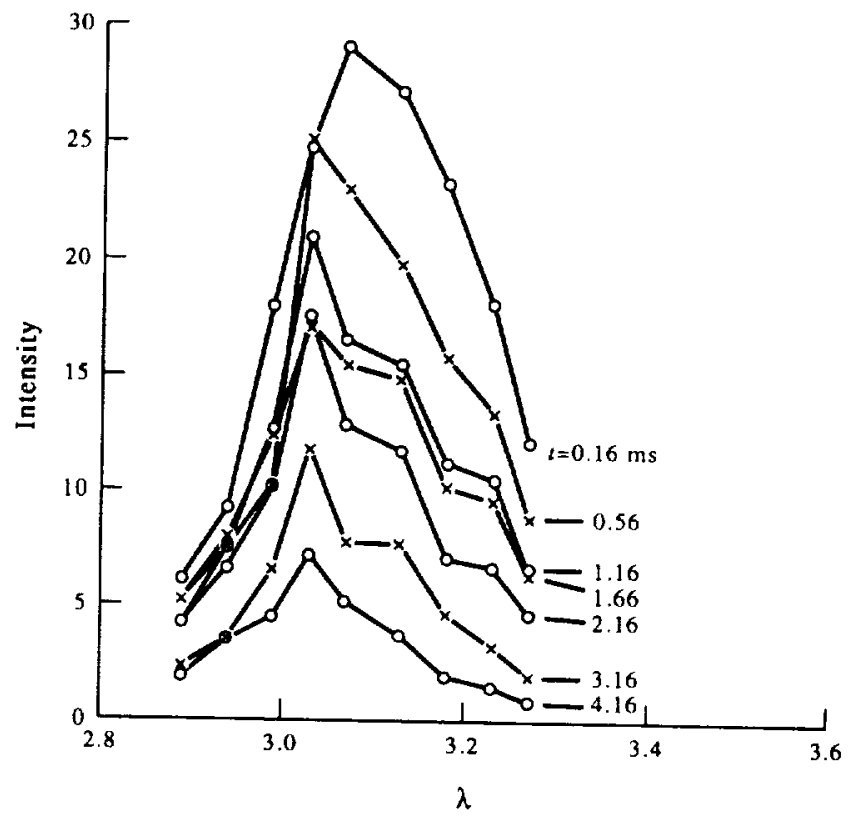

Fig. 7. As Fig. 6, but at a higher shock temperalture $(2650) \mathrm{K})$ 


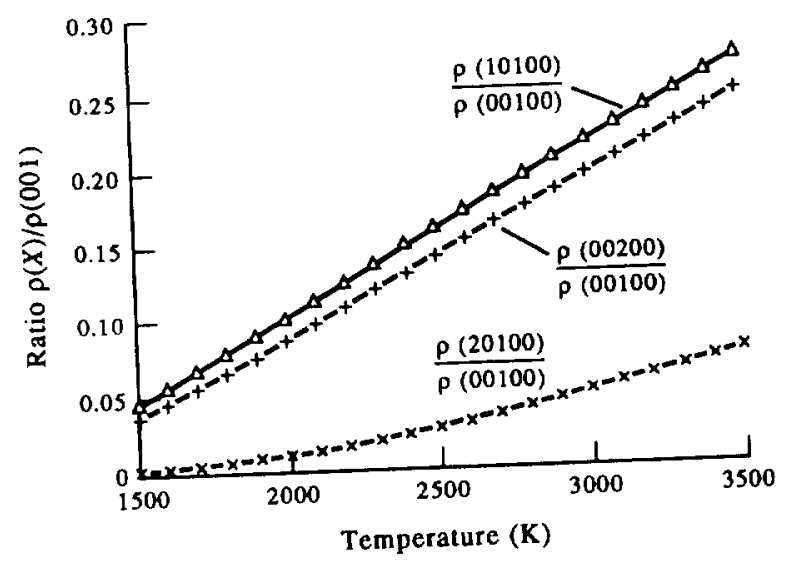

Fig. 8 . Anticipated relative emission intensities for allowed transitions in acelylene. The curves show relative statc populations.

energy of $89.6 \mathrm{kcal} / \mathrm{mol}\left[t_{1 / 2}(1450 \mathrm{~K}) \approx 12.2 \mu \mathrm{s}\right]$. This was derived by fitting measured compositions of extracted samples to a complex mechanism. A more recent analysis based on radical recombination extrapolations [9] led to a considerably lower activation energy, $73.0 \mathrm{kcal} / \mathrm{mol}\left[t_{12}(1450 \mathrm{~K}) \approx 6.9 \mu \mathrm{s}\right]$. Our recorded IR emissions at $1400 \mathrm{~K}$ (Fig. (he isobutene remain for $\approx 1.5 \mathrm{~ms}$, and that the product generated at higher temperatures is acetylene, which radiates at its own characteristic $\mathrm{C}-\mathrm{H}$ frequency.<smiles>C=C(C)C(=C)C</smiles>

In general respects, the thermal emission from 2,3-dimethyl-1,3-butadiene is simiar to that of isobutene, but there are interesting differences. First, note that in Fig. 10 the split between the higher intensity band at $3.4 \mu \mathrm{m}$ is quite distinct, which reflects the relatively large split observed when the corresponding bands were recorded at room temperature in absorption. The time-temperature dependence is more striking. This is illustrated qualitatively in the sequence of oscilloscope traces, Fig. $11\left(T_{5}=925 ; 1110 ; 1574 ; 1873\right.$;

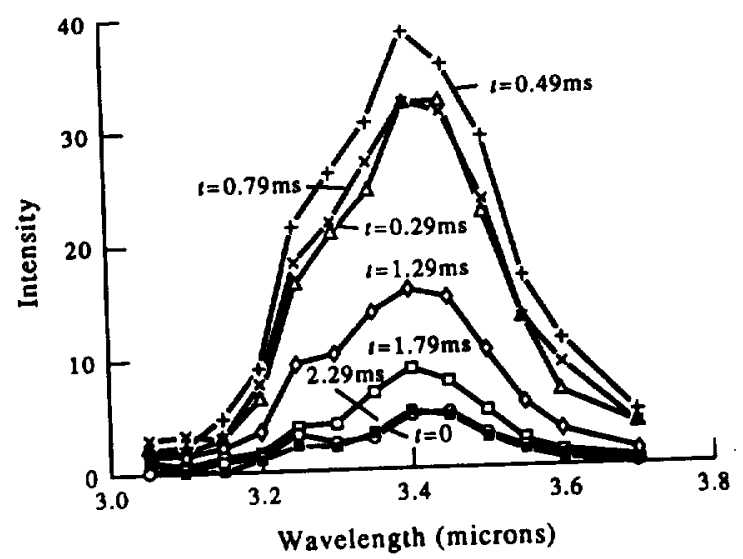

Fig. 9. Time-dependent emission intensities at selected wavelengths from shock healted (CH $\mathrm{C}=\mathrm{CH}(1 \%$ in $\mathrm{Ar})$. at $\approx 1400) \mathrm{K}$. As in Fig. 6, the times shown are post arrival of the reflected shock ... at (3. Fig. 2(b). 


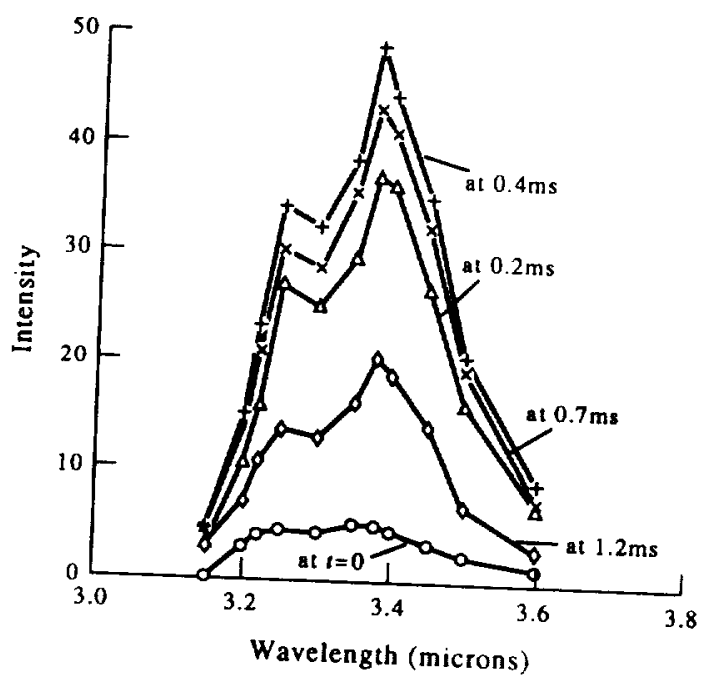

Fig. 10. As Fig. 9, for $1 \% \mathrm{H}_{2} \mathrm{C}=\underset{\mathrm{C}}{\mathrm{C}}-\stackrel{\mathrm{CH}_{3}}{\mathrm{C}}=\mathrm{CH}_{2}$ in $\mathrm{Ar}$, at $\approx 1350 \mathrm{~K}$.

$2555 ; 3685 \mathrm{~K}$ ). At about $1574 \mathrm{~K}$, there is clear indication that the decay at $1.25 \mathrm{~ms}$ is no
longer uniform, providing a hint appears more clearly at $1873 \mathrm{~K}$. When reflected from a newly generated species. This second emission dominated; then both dectined shock temperatures reached $2555 \mathrm{~K}$ the presume that at the higher temperatures a strong higher temperature $(3685 \mathrm{~K})$. We acetylene, but this was not directly recorded. Figure 12 shom appears at $3.05 \mu \mathrm{m}$, due to (corrected for sample density) for a range of reflected 12 shows the evolution of intensities

We found no reports on the pyrolysis of 23 dimock temperatures.

TRENWITH [10a] did measure the fragmentation 2,3 -dimethyl-1,3-butadiene. However, $\left(\mathrm{H}_{2} \mathrm{C}=\mathrm{CH}-\mathrm{CH}=\mathrm{CH}-\mathrm{CH}_{2} \mathrm{CH}_{3} \rightarrow \dot{\mathrm{C}} \mathrm{H}_{3}+\mathrm{H}_{2} \mathrm{C}=\mathrm{CH}\right.$ $(694-759 \mathrm{~K})$. His rate constant, extrapolated to $\left.1574 \mathrm{~K}-\dot{\mathrm{C}} \mathrm{H}_{2}\right)$ in a flow reactor radicals from 3-methyl-penta-1,4-diene occurs with a $1574 \mathrm{~K}$ is $5 \times 10^{\circ} / \mathrm{s}$. Loss of methyl $\left(k_{\mathrm{u}} \sim 2 \times 10^{\mathrm{h}} / \mathrm{s}\right.$ at $\left.1574 \mathrm{~K}[10 \mathrm{~b}]\right)$. The diene structure is tation, as indicated by our recorded emission sequence at 3.40 in the initial fragmen-<smiles>C=C(C)C=C(C)C</smiles>

In most respects the thermal emission from 2,4-dimentyl-1,3-pentadiene is like that from the alkylated butadiene except that a split between the high and low frequency the main band, at $3.37 \mu \mathrm{m}$. Considering there is a shoulder on the high frequency side of band in the absorption spectrum it is not surprive weakness of the shorter wavelength corresponding emission as a separate feature. Figures that we could not record the to Figs 10 and 11 , and are self-explanatory. Figures 13 and 14 present data analogous

It is evident that pentadiene, as does

species that emits at the same $\mathrm{C}-\mathrm{H}$ frequency $( \pm 0.04$, pyrolyzes to generate another a maximum at $0.75 \mathrm{~ms}$ (measured from the $( \pm 0.04 \mu \mathrm{m})$. Initially the intensity rises to Fig. 2), then falls and rises to a second the time the reflected shock reaches sensor $7 \mathrm{~b}$, in This is direct evidence that the pyrolysis product is an at the higher temperatures. basis of the available emission spectra one can only speculate alkylated alkene. On the emitters that appear for reflected shock temperaturesulate on the identity of the late 

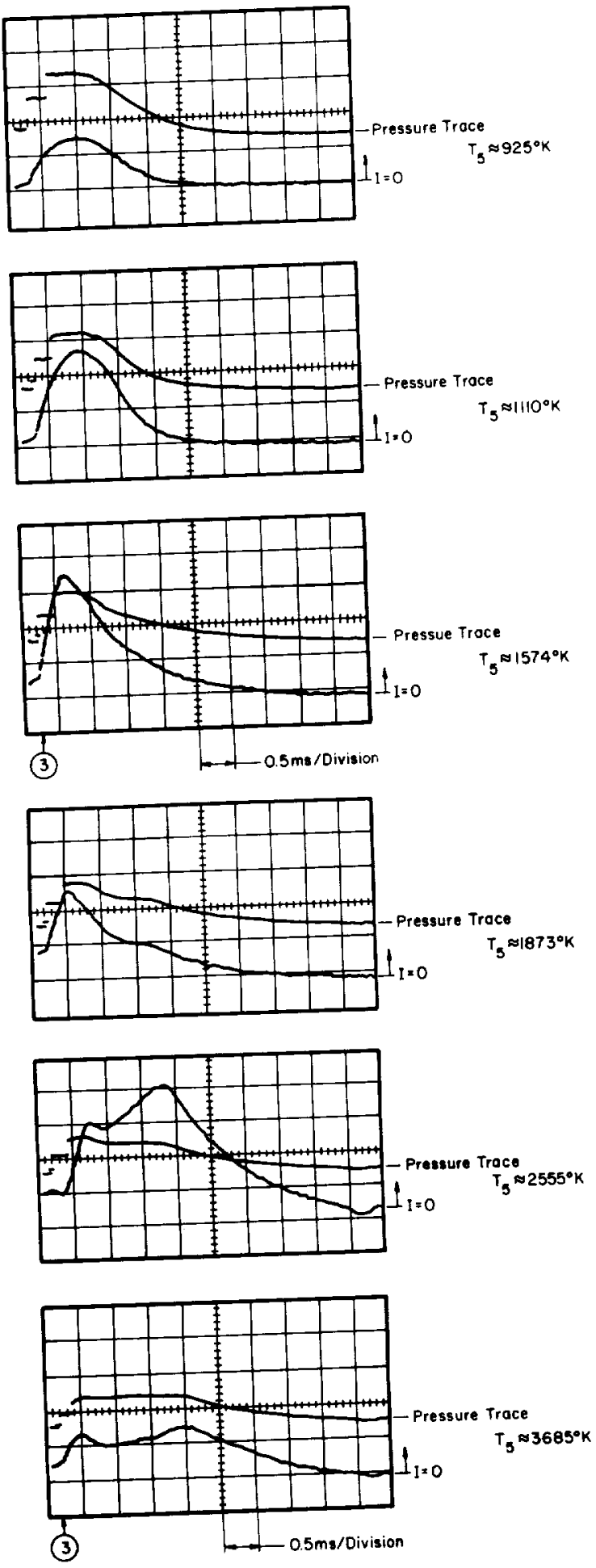

Fig. 11. Temporal emission profiles from<smiles>C=C(C)C(=C)C</smiles>

$\mathrm{CH}_{3}$

and relative intensities for a sequence of reflected shock temperatures showing pressure traces and relative intensics for al sequence The development of a derivative $\left(T_{5} \approx 925 ; 1110 ; 1574 ; 1873 ; 2555:\right.$ and $\left.3685 \mathrm{~K}\right)$ at $3.40 \mu \mathrm{m}$. The development of a derivative species (with increasing temperature) that emits at th 


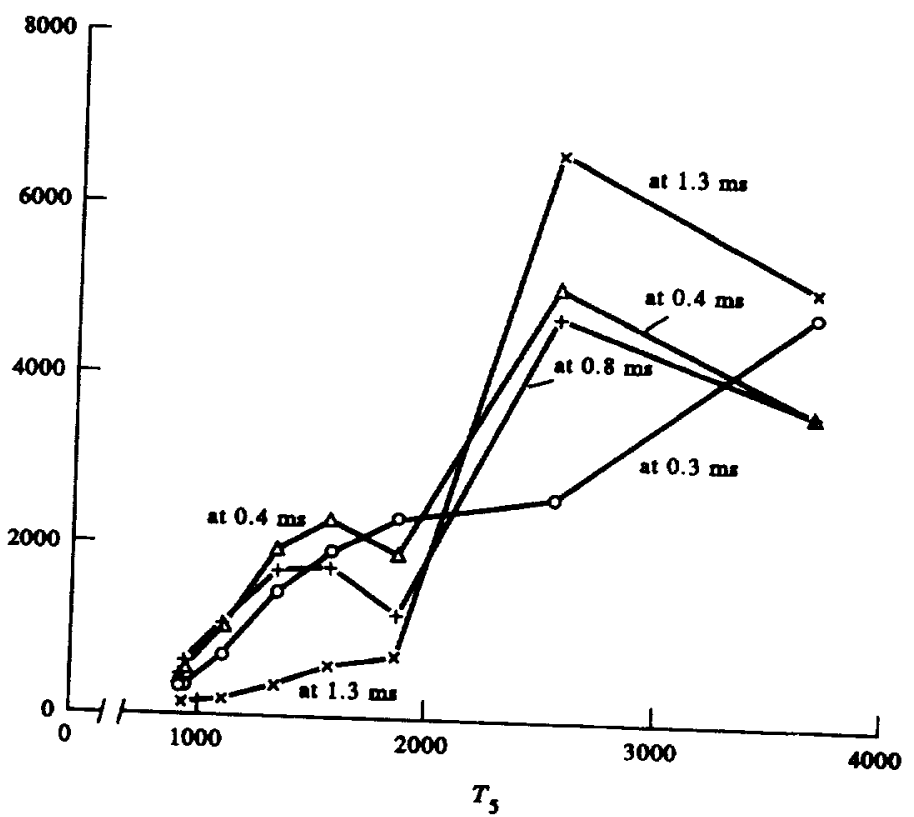
Fig. 12. Temperature dependence of emission intensities at various times after shock arrival at
positon (1) Fig. 2 (b), for<smiles>C=C(C)C(=C)C</smiles>

recorded at $3.36 \mu \mathrm{m}$. These intensities were corrected for sample density.

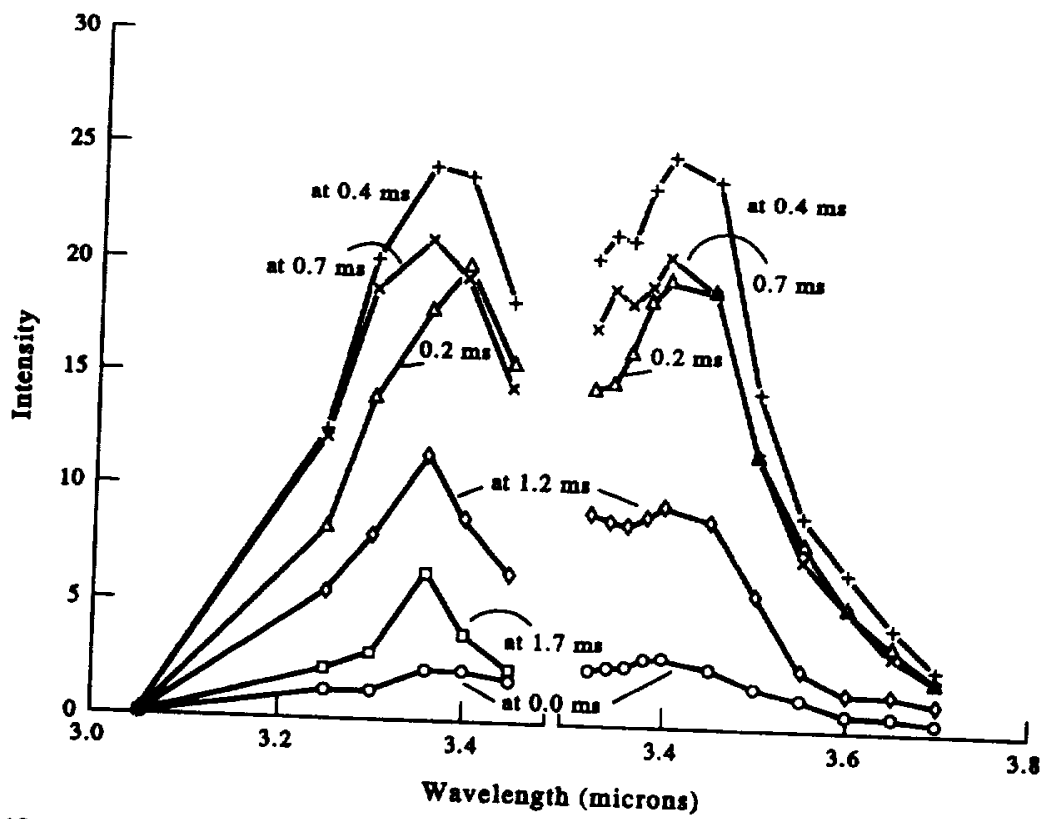

Fig. 13. Emission intensities at a sequence of wavelengths from shock-heated<smiles>C=C(C)C=C(C)C</smiles>

at $T_{5} \approx 1360 \mathrm{~K}$. Two independent sets of

of reproducibility. Times measured post-reflected displaced, and presented to indicate degrees 


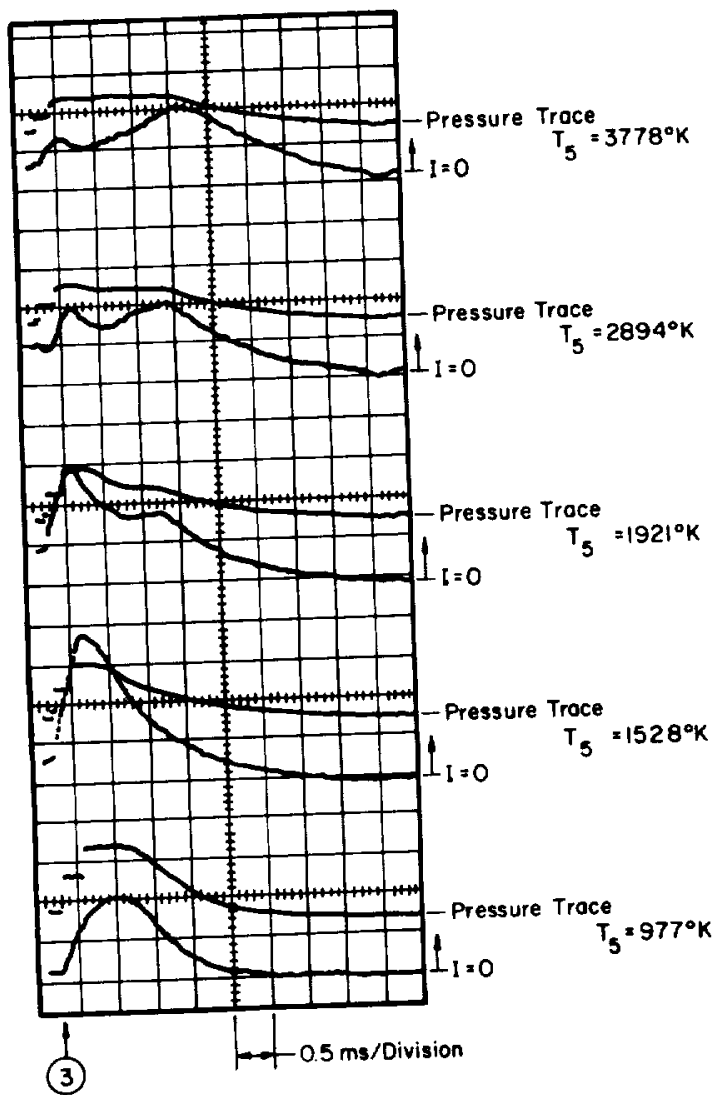

Fig. 14. Temporal emission profiles (as in Fig. 11) for<smiles>C=C(C)C=C(C)C</smiles>
for a range of shock temperatures $\left(T_{5}=977 ; 1528 ; 1921 ; 2894 ;\right.$ and $\left.3778 \mathrm{~K}\right)$, at $3.36 \mu \mathrm{m}$.

post-shock reflection, then grow to a maximum at $\approx 1.7 \mathrm{~ms}$ and finally decay. In each case the initial conversion involves $\mathrm{C}-\mathrm{C}$ bond breaking, to generate free radicals. These rapidly rearrange and react to produce a host of smaller species that incorporate $=\mathrm{CH}_{2}$ units. On the time scale of several milliseconds, at shock-tube temperatures and densities, the ensemble has relaxed substantially, but not completely, toward an equilibrium compositon. The partition of $\mathrm{C} / \mathrm{H}$ products (at equilibrium) for systems with $\mathrm{C} / \mathrm{H} \approx 1 / 2$ was calculated [11]. At $T=1500 \mathrm{~K}$ the major components are $\mathrm{C}_{2} \mathrm{H}_{2}$ and $\mathrm{C}_{6} \mathrm{H}_{6}$. However, benzene disappears rapidly at $T>1600 \mathrm{~K}$. The major remaining species are $\mathrm{C}_{4} \mathrm{H}_{2}$ and $\mathrm{C}_{2} \mathrm{H}_{4}$. It is possible that higher resolution spectra would permit identification of these late emitters.

\section{Critioue of the PAH Hypothesis}

An excellent review of interstellar emission features in the IR, covering both spectral data and a variety of assignments thus far proposed, was prepared by SELLGREN [1]. She summarized an extensive literature, which continues to evolve at a high rate. Her Table 2 is a compilation of the principal recorded features $(3.3,6.2,7.7,8.6,11.3 \mu \mathrm{m})$, the molecular frequencies that correspond (approximately) to these bands, and their possible astrophysical sources. The latter include: AC (amorphous carbon grains); coal (vitrinite grains); HAC (hydrogenated amorphous carbon); orgueil (carbonaceous residue from meteorites); QCC (quenched carbonaceous composites); and PAH (polycyclic 
aromatic hydrocarbons). A more extended review was presented by ALLAMANDOLA et al. [12]. It covered the chemistry, IR emission theory, spectroscopy and astrophysical applications of the PAH hypothesis.* At this stage it appears that this model has gained discovered that matches all is clear that no single Earth-bound substance has been concurrently does not indicate observed features in wavelength and intensity, and attempts to unravel the mystional strong features, which are not observed. In the many aspects treated in the of $\mathrm{U}$ emissions one should consider in detail (a) whatever the proposed species, the reviews, and focus on the following three: production in the interstellar medium: (b) it should be chemically plausible route for its mentally the survival of exibsible to demonstrate expericlosely resemble the remitters, at least for milliseconds; (c) their IR emissions should

recorded astronomical spectra.

are carbon rich objects radius, heated to about $1000 \mathrm{~K}$, with the suggestion that grains of approximately $10 \AA$ from reflected nebulae. It is wore required to explain the near-IR continuum emissions the emitters are relatively low moting that in neither review was the possibility that sidered. However, the best astron species connot rule out molecular band emissions fesolution currently available $(\lambda / \Delta \lambda \approx 1600$ does FTIR spectrometers $\left(2 \mathrm{~cm}^{-1}\right.$ resolution), focies in the class $\mathrm{C}_{n} \mathrm{H}_{m}$, with $n \geqslant 5$. Standard relatively broad band profiles for methyl, equivalent to $(\lambda / \Delta \lambda \approx 1300)$ record smooth, dimethyl-1,3-butadiene. Indeed, it was propnized that the cambl-1,3-pentadiene and 2,3PAHs generally fail to match the interstecosnized that the calculated emission spectra of related dienes show absorption spectra (Fig. 1) that match, whereas a mix of closely better than any source proposed, including. 1) that match overall the UIR features other listed emitters, these call for an the troublesome $7.7 \mu \mathrm{m}$ region; but like all Perhaps, because of its high absorption unobserved band (at approximately $7.0 \mu \mathrm{m}$ ). low due to self-absorption by a cooler coefficient, the recorded intensity at $7.0 \mu \mathrm{m}$ is medium.

Since the UIR bands are associated with reg UV radiation, any proposed IR emitter whith regions subjected to considerable fluxes of for its formation but also for its emitter should not only comply with some mechanism the ground electronic state; see EuRENFre radiating from excited vibrational states in [15] calculated that PAH species could be carbon stars, provided: (i) the ambient generated in stellar winds from red giant (ii) these remain in hot regions for thous levels of acetylene were particularly high; winds involved are dense and slow. This mechan at $90(0-1100 \mathrm{~K}$; and (iii) the stellar

With respect to mechanisms for This mechanism was reviewed and extended [12]. significant problems that have not been adequand survival of PAH species, there are and Allamandola et al. [17]. proposed thatequately addressed. LÉGER and PUget [16], the PAH species, by internal conversion, lodge in electronic state and emit in the IR by seqeuntial $\Delta v=-1$ transitions. This mechanism
requires that the bands should be degraded toward $\mathrm{C}-\mathrm{H}$ stretch vibrations, due to their high anharmard longer wavelengths, particularly for call attention to this difficulty. The calculated emisicities. Allamandola et al. [12] did $3.3 \mu \mathrm{m}$ region does show satellite peaks at $3.43 \mu \mathrm{m}(\nu=2 \rightarrow 1)$ and $3.57 \mu \mathrm{me}$ [18] in the with intensities that are strongly dependent on the $(\nu=2 \rightarrow 1)$ and $3.57 \mu \mathrm{m}(\nu=3 \rightarrow 2)$, Low level emissions, roughly at these frequencies, assumed vibrational energy content. NGC7027.

LÉGER et al. [19] and JOURDAIN DE MUIZON et al. [20] provided an extended analysis of their model, based on the assumption that the thermal history of an energized isolated multi-atomic molecule is radiatively equivalent to an ensemble, at an equivalent

\footnotetext{
* The hypothesis that PAHs are formed in red giants has been further anblyzed in Ref. [13]. They calculated molecular ahundance profiles for a wide range of $\mathrm{C} / \mathrm{H}$ species. . . radicals, long chain hydrocarbons and PAHs
in carbon-rich stellar photospheres. Under in carbon-rich stellar photospheres. Under thermal equilibrium conditions long chain hydrocarbons appear
abundant at moderate temperature, but only henzene (among the aromatics) survives grapite condensation.
} 
temperature. Then thermal relaxation can be calculated using statistical mechanics relations [21]. Even though Léger derived several predictions that were later verified, he also called attention to several problems to which his model does not respond. At such high levels of vibrational excitation, dissociation and fragmentation should effectively compete with IR emissions, where the radiative lifetimes are as long as $10^{-3} \mathrm{~s}$. Application of the rudimentary RRK expression by Léger to estimate longer dissociative lifetimes is not valid, as demonstrated experimentally by SCHLAG and LEVINE [22]; and discussed theoretically by Lorouet et al. [23]. SEWFLL et al. [24] showed via classical trajectory calculations, that depending on mode of excitation, some molecular trajectories fail to representatively sample all the available phase space. Such observations place into question the survivability of highly vibrationally excited PAH species for the relatively long lifetimes required for IR emission, as presented in the more sophisticated analysis of this question via the QRRK model [25]. However, recently an analysis was presented [26], based on master equation calculations, that concluded that at low temperatures IR emissions from nascent species are efficient in deactivating and stabilizing adducts of recombination reactions. Consider also that transition moments for electronic excitations are orders of magnitude greater than for vibrational transitions in the IR; one should anticipate spectroscopic signatures of PAH in the visible at $\approx 550 \mathrm{~nm}$ $\left(\mathrm{C}_{41} \mathrm{H}_{21}\right)$, and in the near UV at $\approx 350 \mathrm{~nm}$ [27]. The large levels of PAH currently proposed should make such features readily detectable. In this respect no data are available. Finally, there is an enormous number of isomers for $\approx 100$ atom PAHs $[28,29]$. One should anticipate a significant spread in $\mathrm{C}-\mathrm{H}$ frequencies, were there isomers generated on a random basis. However, most mechanisms that have been proposed for PAH formation do not postulate random isomer distributions.

\section{Conclusions}

When low molecular weight olefines and dienes $\left(\mathrm{C}_{4} \rightarrow \mathrm{C}_{7}\right)$ are shock heated to temperatures above $900 \mathrm{~K}$, their IR emission spectra are reflections of their room temperature absorption spectra. Conversions to other similarly emitting species take place when these are exposed to such elevated temperatures for periods of several milliseconds. We propose that the spectra described in this report present these species as plausible contributing candidates for UIR emitters. It is worth noting that with respect to survivability under UV and visible irradiation, the dienes have bands in the $220 \mathrm{~nm}$ region with $\log \varepsilon \sim 4$, while the polycyclic aromatics have extended absorption bands in the UV and visible, down to $600 \mathrm{~nm}$ [30] with $\log \varepsilon \sim(4 \rightarrow 5.5)$. Relative propensities for dissociation and ionization are not well established for radiation levels present in astronomical media.

Acknowledgements-This study was supported by grants from NSF (Divison of Astronomical Sciences: AST-8704623) and NASA (Astrophysics Division: NAGW-1262).

\section{REFERENCES}

11] K. Sellgren, Dusty Objects in the Universe, p. 35ff. Kluwer Academic Publishers, The Netherlands (1990).

Bauer and D. B. Borchardt, I7th Inlernational Symposium on Shock Waves, p. 600. API

(2) S. H. Bauer and D. B.

13] E. F. Green and J. P. Toennies, Chemical Reactions in Shock Waves. Arnold, London (1964).

14] B. Colket.

[4] M. B. Colket.

[5] Y. Hidaka, H. Masaoka et al., Int. J. Chem. Kin. 24, 871 (1992).

[6] M. S. Child and R. T. Lawton, Farad. Discuss. Chem. Soc (1969).

[7] M. Pathak and W. B. Fletcher, J. Molec. Spectrosc. 31, S2 (19arday Trans. I 72. 558 (1976).

19] A. M. Dean, J. Phys. Chem. 89, 4600 (1985).

[10] (a) A. B. Trenwith, J. Chem. Soc. Faraday Trans. 176, 266 (1980); (b) A. B. Trenwith, J. Chem. Soc. Faraday Trans. I 78, 3131 (1982).

111] R. E. Duff and S. H. Bauer, J. Chem. Phys. 36, 1754 (1962).

(12) L. J. Allamandola, A. G. G. M. Tielens and J. R. Barker, in Interstellar Processes (Edited hy D. J. Hollenbeck and H. A. Thronson). Reidel. Dordrecht (1987).

[13] I. Cherckneff and J. R. Barker. Astrophys. J. 394. 703 (1992). 
[14] P. Ehrenfreund, F. Robert. L. dHendecourt and F. Behar. Astron. Astrophys. 252, 712 (1991)
[15] M. Frenklach and E. D. Feigelson, Astrophys. J. 341. 372 (1989).

[16] A. Léger and J. L. Puget, Astron. Astrophys. 137, L.5-L8 (1484)

[17] L. J. Allamandola. A. G. G. M. Tielens and J. R. Barker, Astrophys. J. 290, L25 (1985).

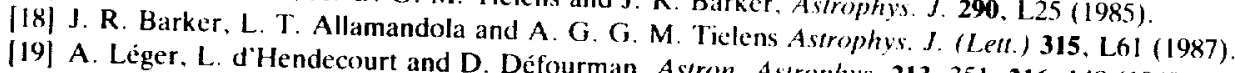

[20] M. Jourdain de Muizon, L. B. d'Hendecourt and T R. Astrophys. 213. 351; 216, 148 (1989).

121) R. C. Dunbar, J. Chem. Phys. 90.7369 (1989).

[22] E. W. Schliag and R. D Levine,

[23] J. C. Lorquet J M. E. Levine, Chem. Phys. Lett. 163, 523 (1989)

and R. D. Levine, Chem. Phys. Le'll. 175, $461(1990)$

[25] L. T. Allamandola, A Gompson and R. D. Levine, J. Phys. (hem. 96, 806)6 (1992)

[26] J. R. Barker, J. Phys. Chem. 96.7361 (1992). Barker, Astrophys. I. Supp. Series 71, 733 (1989).

[27] J. C. Fetzer Polynur

[28] J. R. Dias, J. Chem. Inf. Comp. 22, 139 (1982)

[29] S. J. Cyvin and J. Brunvall, Chem. Phys. Lett. 170, 364 ( 1990 )

[30] W. Karacher et al., Spectral Atlas of Polycyclic Aromatic (simp (1983).

\section{Appendix 1: Comments on Time-Proflles}

In this study, all the emission spectra were recorded from samples in the reflected shock regime. Then the initially heated gas (via the incident shock) remains essentially progresses away from the the forward section of the tube, as the reflected shock diagram in Fig. 2(b) sample mindow, and reheats the sample (to $T_{5}$ ). [In the $x / t$ emission intensity traces show that is indicated by dashed lines.] The oscilloscope higher temperature runs, were gent some low intensity emissions, particularly for the the present analysis we treat this as "backgre incident shock, as was anticipated. In profiles were not recorded. The buildup anround radiation", since those time/intensity integrated output due to: (i) the growing decay of intensity for $t>(3)$ Fig. 2(b) is the superposed (ii) the time-dependent excitatiopth of the reheated gas, upon which is within any selected slab of material bitation and decay mechanism for the emitters reflecting window. There is an additional between $x$ and $(x+\Delta x)$, measured from the light collection efficiency, $g(x)$. Because correction factor for the $x$ dependence of the smooth, it functions as a

$$
g(x) \approx a+b \exp (-c x) \text {, with } a=0.88 ; b=0.12 ; c=0.20\left(\mathrm{~cm}^{-1}\right) .
$$

Thus, from any slab, the collected intensity is

$$
\begin{array}{ll}
l_{1}(t)=g(x) \cdot E\left(t-x / u_{5}\right) \cdot \Delta x, & \text { for } t>x / u_{5} \\
A \stackrel{k_{1}}{\rightarrow} A^{*} & \text { (excitation by thermal collisions) } \\
A^{*} \stackrel{k_{2}}{\rightarrow} B & \text { (loss of pyrolysis) } \\
A^{*} \stackrel{k_{3}}{\rightarrow} A+\phi & \text { (radiation). }
\end{array}
$$

This leads to a characteristic double exponential solution:

$$
\begin{aligned}
\frac{1}{A_{11}} \frac{\mathrm{d} \phi}{\mathrm{d} t} & =k_{3}\left(A^{*}\right)=\frac{k_{3} \cdot k_{1}}{\left(k_{2}+k_{3}\right)-k_{1}}\left[\mathrm{e}^{-k_{1}\left(t-1 / t_{5}\right)}-\mathrm{e}^{\left(k_{2}+k_{2}\right)\left(t-v^{\prime} / t_{3}\right)}\right] \\
& =\frac{k_{3} \cdot k_{1}}{\left(k_{2}+k_{3}\right)-k_{1}} \exp \left[-\left(k_{2}+k_{3}\right) t\right]\left\{\mathrm{e}^{-\left(k_{1}-k_{2}-k_{3}\right) \prime} \mathrm{e}^{k_{1} / u_{4}}-\mathrm{e}^{\left(k_{2}+k_{3}\right) / t_{5}}\right\},
\end{aligned}
$$

and the overall time profile is:

$$
I_{5}(t)=\frac{k_{3}}{A_{4}} \int_{t=11}^{x_{4}} g(x) \cdot\left(A^{*}\right) \mathrm{d} x
$$

At $1500 \mathrm{~K}$, estimated orders of magnitude: $k_{1} \approx 10^{7} ; k_{2} \approx 10^{5} ;$ and $k_{3} \approx 10^{3} \mathrm{~s}^{-1}$.

Qualitatively the data do appear to follow the double exponential form for the lower temperature shocks, but require two sets of double exponentials to account for emission intensities recorded for the higher temperature runs $(T>1600 \mathrm{~K})$. 